\title{
Analytical Evaluation of the Performance of Contact-Based Messaging Applications ${ }^{\not 3}$
}

\author{
Enrique Hernández-Orallo ${ }^{\mathrm{a}}$, Marina Murillo-Arcila ${ }^{\mathrm{b}}$, Carlos T. Calafate ${ }^{\mathrm{a}}$, Juan \\ Carlos Cano $^{\mathrm{a}}$, J. Alberto Conejero ${ }^{\mathrm{c}}$, Pietro Manzoni ${ }^{\mathrm{a}, *}$ \\ ${ }^{a}$ Departamento de Informática de Sistemas y Computadores. Universitat Politècnica de \\ València. Spain. \\ ${ }^{b} B C A M$ - Basque Center for Applied Mathematics. Spain. \\ ${ }^{c}$ Instituto Universitario de Matemática Pura y Aplicada. Universitat Politècnica de \\ València. Spain.
}

\begin{abstract}
Communications in mobile opportunistic networks, instead of using the Internet infrastructure, take place upon the establishment of ephemeral contacts among mobile nodes using direct communication. In this paper, we analytically model the performance of mobile opportunistic networks for contact-based messaging applications in city squares or gathering points, a key challenging topic that is required for the effective design of novel services. We take into account several social aspects such as: the density of people, the dynamic of people arriving and leaving a place, the size of the messages and the duration of the contacts. We base our models on Population Processes, an approach commonly used to represent the dynamics of biological populations. We study their stable equilibrium points and obtain analytical expressions for their resolution.

The evaluations performed show that these models can reproduce the dynamics of message diffusion applications. We demonstrate that when the density of people increases, the effectiveness of the diffusion is improved. Regarding the arrival and departure of people, their impact is more relevant when the density of people is low. Finally, we prove that for large message sizes the effectiveness of the epidemic diffusion is reduced, and novel diffusion protocols should be considered.
\end{abstract}

Keywords: Opportunistic networks, Mobile Networking in Proximity, Contact-based Messaging, Performance Evaluation, Epidemic diffusion

\footnotetext{
This work was partially supported by Ministerio de Economia y Competitividad, Spain (Grants TEC2014-52690-R \& MTM2013-47093-P \& BCAM Severo Ochoa excellence accreditation SEV-2013-0323), Generalitat Valenciana, Spain (Grants AICO/2015/113 \& ACOMP/2015/005) and by the Basque Government through the BERC 2014-2017 program.

* Corresponding author.

Email addresses: ehernandez@disca.upv.es (Enrique Hernández-Orallo), mmurillo@bcamath.org (Marina Murillo-Arcila), calafate@disca.upv.es (Carlos T. Calafate), jucano@disca.upv.es (Juan Carlos Cano), aconejero@upv.es (J. Alberto Conejero ), pmanzoni@disca.upv.es (Pietro Manzoni)
} 


\section{Introduction}

The authors in [1] define Mobile Social Networking in Proximity (MSNP), as a wireless peer-to-peer network of opportunistically connected nodes that use proximity as the social relationship. This condition allows the establishment

5 of local communication channels that can be used for applications such as information sharing, advertisement, disaster and rescue operations, gaming, etc. Instead of using the established Internet infrastructure, the communication in mobile opportunistic networks takes place upon the establishment of ephemeral contacts among mobile nodes using direct communication (i.e. Bluetooth or

10 WiFi Direct). Moreover, by relying on properly designed security mechanisms, mobile opportunistic networks can increase the confidentiality and privacy of communications, since direct communications, unlike infrastructure based communication, are more robust to the tracking of the user behaviour.

Based on the concept of opportunistic networks, new contact-based messaging applications have recently been developed. Firechat, as an example, a messaging application meant for festivals, became popular in 2014 in Iraq due to the government restrictions on Internet us $\AA^{1}$, and after that during the Hong Kong protest: ${ }^{2}$. There are anyway other examples, like Briar (see https://briarproject.org) which is a secure messaging application, or CoCam

20 2] for image sharing in events. Moreover, several supporting frameworks and architectures are appearing, such as the Haggle project [3, a framework for autonomic and opportunistic computing, or AllJoyn, an open source, general networking framework from the Allseen Alliance (https://allseenalliance.org/). The experience shows that these messaging applications seem to be operative 25 in open places with a moderate to high density of people.

In this paper, we analytically study the performance of these opportunistic contact-based messaging strategies in city squares or gathering points. According to a recent survey 4 analytical modelling and performance evaluation of DTNs and Opportunistic Networks is one of the key challenging problems. A

30 common approach is to combine a network simulation tool with realistic mobility traces. Nevertheless, simulation can be very time consuming and restricted to the limited scenarios of the available mobility traces. Analytical models can avoid these drawbacks providing a fast and broader performance evaluation. Two classes of analytical models have been proposed for modelling this network 35 dynamics: Markovian models [5, 6, 7, 8, 9, 10] and deterministic models based on Ordinary Differential Equations (ODEs) [6, 11, 12, 13. Analytical models require anyway a precise and concise description of the mobility scenario, that

\footnotetext{
${ }^{1}$ Kuchler, Hannah; Kerr, Simon. "Private Internet: FireChat app grows in popularity in Iraq". Financial Times, 2014-06-22

${ }^{2}$ Bland, Archie. "FireChat the messaging app that's powering the Hong Kong protests". The Guardian, 2014-09-29.
} 
usually assume that the inter-contact times distribution between pairs of nodes are exponentially distributed with a given contact rate 5 .

$40 \quad$ We based our model on Population Processes, a method commonly used to model the dynamics of biological population 14. More specifically to opportunistic networks, Haas and Small [6] presented a model based on epidemiological processes for a network that used animals (whales) as data carriers to store and transfer messages (an approach similar to DTN). Zhang et al. [11] derived

45 ODE equations for the study of the dynamics of various forwarding and recovery DTN schemes, such as epidemic and 2-hop, among others. The authors of 12 introduced a mathematical approach for messages diffusion in opportunistic networks using the Epidemic protocol. This approach is based on well known models for the spreading of human epidemical diseases, e.g. SIR (Susceptible, 50 Infectious and Recovered) models. One of the main conclusions of their analysis (mathematical model and its respective simulation) is that SIR models are quite accurate for the average behaviour of Epidemical DTN.

In [13] the authors propose a detailed analytical model to analyse the epidemic information dissemination in mobile social networks. It is also based on

55 SIR models including rules that concern user's behaviour, especially when their interests change according to the information type, and it can have a considerable impact on the dissemination process. After large simulations, they have demonstrated the accuracy of their model.

Nevertheless, these previous models, do not take into account several social ${ }_{60}$ aspects that impact the performance of message dissemination such as: the density of people, the dynamic of people arriving and leaving a place, the size of the messages and the duration of the contacts. We therefore introduce dynamic models that take into account these social aspects, based on Delay Differential Equations (DDEs). DDEs are similar to Ordinary Differential Equation

65 (ODEs), but their evolution involves past values of the state variable. DDEs have been used for modelling population dynamics in many disciplines such as biology, ecology, epidemics [15], and network protocol analysis [16].

For these models we studied their stable equilibrium points and obtained analytical expressions for their resolution. The evaluations performed showed

70 that these models can reproduce the dynamics of message diffusion. We show that when the density of people increases, the effectiveness of the diffusion is improved. Regarding the arrival and departure of people, the impact is more important when the density of people is low. Finally, we prove that for large message sizes the effectiveness of the epidemic diffusion is reduced, and novel diffusion protocols should be considered.

The paper is organised as follows. Section 2 describes the contact-based messaging dissemination approach that we assume. Section 3 presents the models and Section 4 the evaluation of a few scenarios using our approach. Finally Section 5 offers the conclusions. 


\section{The considered contact-based messaging approach}

The class of contact-based messaging applications we are considering in this work is based on establishing a short-range communication directly between mobile devices, and on storing the messages in these devices to achieve their full dissemination. It can be considered a wireless peer-to-peer $(\mathrm{P} 2 \mathrm{P})$ network of nodes that connect opportunistically. No messages are sent or stored in servers, rather, all information is stored on the mobile devices in a given area, i.e., it can be seen as a localised approach instead of a cloud approach.

Message spreading is based on epidemic diffusion, a concept similar to the spreading of infectious diseases, where an infected node (the one that has a

90 message) contacts another node to infect it (transmit the message). Epidemic routing obtains the minimum delivery delay at the expense of increased usage of local buffer and increased number of transmissions. There are different variations of this diffusion scheme (that is, the infection process), that attempt to reduce resource utilisation e.g., 2-hop forwarding [5], probabilistic forwarding 17] and spray-and-wait or multiple copy [18, 7].

The diffusion of messages among users is organised in groups. Users can join (and leave) a group, and their members receive the messages that are sent to the group. Thus, the goal is to spread all the messages of the various groups so that they can be received by the subscribed members. The mechanisms for group management are, anyway, outside the scope of this paper.

Message dissemination takes place as follows. Mobile devices have a messaging application that notifies and shows the user the received messages for the subscribed groups. Each node has a limited buffer where the messages in transit can be stored. When two nodes establish a pair-wise connection, they exchange the messages they have in their buffer, and check whether some of the newly received messages are suitable for notification to the user. All nodes that have the messaging application collaborate in storing and forwarding messages.

One important condition that must hold is that the contact between two nodes should last long enough for transferring the whole message. This condition holds true depending on the application behaviour when two nodes contact. Two approaches can be assumed:

- The devices stop when they need to exchange information. In this case, the owners of the mobile devices control this exchange by waiting until the message transmission is completed. This is a commonly used scheme in several existing short-range messaging protocols such as Apple iOS Airdrop and Google Android Copresence.

- The devices do not stop, so the completion of message transmission will depend on the contact duration. In this case, when the contact duration is lower than the message transmission time, the transmission fails. The failure probability will clearly depend on the users mean speed and communication range. For example, if nodes are expected to be constantly moving at a mean speed of $0.5 \mathrm{~m} / \mathrm{sec}$ and communication range is $7.5 \mathrm{~m}$, 
a rough estimation (it depends on the direction of the devices) of the average contact duration time would be approximately $7.5 * 2 / 0.5=30 \mathrm{sec}$, that is not enough time, for example, to transfer a $4 \mathrm{MB}$ photo at $1 \mathrm{Mbps}$.

In our paper, we assume the first approach, and therefore suppose that user control the devices stop time.

\section{Performance model}

In this section we propose an analytical performance model to evaluate the

130 where people can enter and leave. The performance model presented here is based on population processes or models. A population model is a type of mathematical model which is applied in order to study population dynamics, and it is extensively used to model biological population such as the spread of para-

135 sites, viruses, and diseases [14. Specifically, our model is based on biological epidemic models [19, where individuals can be infected when a contact occurs with other infected nodes. In our case, an infected node refers to a node that has a message, and an infection, when a node that has a message transmits it to another node. Nodes move freely in a given area with a given contact rate leave the place with an exit rate of $\delta$. Thus, the number of nodes (population) in the place $(N)$ depends on the initial number of nodes in the place $\left(N_{0}\right)$ and the rates of arrival and exit. We assume a short-range communication range (for example, Bluetooth), so network congestion and interferences do not have a strong impact.

First, we present a basic epidemic model (Section 3.1), well known in the literature that we later extend (Section 3.2), with the arrival and exit rates in order to evaluate in depth its dynamics and stability. The last model (Section 3.3 will take into account the peer-to-peer communication delay.

\subsection{Basic epidemic model}

In the basic epidemic model [11, the number of nodes in a place remains constant, and when a node carrying the message (an infected node) contacts with another node that does not have the message (called the susceptible node) it transmits this message immediately. From that moment on, both nodes carry

155 the message. As we will prove, this model is valid only when the transmission time is low.

Following the epidemic model notation, the population is divided into two classes: the infected nodes $(I)$ and the susceptible nodes $(S)$, and population remains constant: $N=N_{0}=I+S$. Thus, there is only one possible transition from class $S$ to $I$ that occurs when a susceptible node is infected, i.e., nodes move from class $S$ to $I$ with rate $\lambda S I:(S \rightarrow I, \lambda S I)$. The dynamics of this system can be expressed using a deterministic model based on ODEs (Ordinary 
Differential Equations):

$$
\begin{aligned}
S^{\prime}(t) & =-\lambda S(t) I(t) \\
I^{\prime}(t) & =\lambda S(t) I(t)
\end{aligned}
$$

These ODEs have an analytical solution assuming one initial node infected $(I(0)=1)$ [11. More specifically, the function that represents the infected nodes is the logistic function:

$$
I(t)=\frac{N}{1+(N-1) e^{-\lambda N t}}
$$

Using the previous defined transition rates, we could also derive a Continuous Time Markov Chain (CTMC). Although Markovian models can provide more complex metrics (such as complete distributions), the numerical solution of these models becomes unfeasible when the number of nodes is large (as occurs in the scenarios evaluated in this paper). On the contrary, as populations $\mathrm{N}$ grows large, ODEs can be derived as limits of these Markovian models, as stated by Kurtz's convergence theorem [20].

\subsection{Epidemic model for an open area}

165 In this Section, we extend the previous model to take into account that nodes can enter and leave an open area (e.g., a public square, a shopping mall, etc.). The arrival rate is $\beta$, and it is equivalent to the birth rate of the epidemical models. We assume that a newly arrived node is a susceptible node (it does not have the message). The exit rate is $\delta$, and both susceptible and infected nodes can leave the area. This differs from the typical death rate of epidemical models, where only the infected nodes can die (that is, leave the place). Finally, the final exit rate in both classes is proportional to the relative number of susceptible and infected nodes. Thus, the number of nodes is not constant along time and can be obtained as $N(t)=N_{0}+(\beta-\delta) t$ where $N(t)=I(t)+S(t)$ and $N(t)>0$. Summing up, the system has the following transitions:

- $(\rightarrow S, \beta)$ : new nodes enter the place with $\beta$ rate.

- $(S \rightarrow I, \lambda S I)$ : new nodes get the message when contacts occurs.

- $(S \rightarrow, \delta S /(I+S))$ : nodes with no message leave the place.

- $(I \rightarrow, \delta I /(I+S))$ : nodes with the message leave the place

and the dynamics of this system can be expressed using a deterministic model based on ODEs:

$$
\begin{aligned}
S^{\prime}(t) & =-\lambda S(t) I(t)+\beta-\delta S(t) / N(t) \\
I^{\prime}(t) & =\lambda S(t) I(t)-\delta I(t) / N(t) \\
N^{\prime}(t) & =\beta-\delta
\end{aligned}
$$

Figure 1 shows the evolution of the infected nodes $(I(t))$ and the number of nodes $(N(t))$ as a function of time for different values of the arrival and exit 


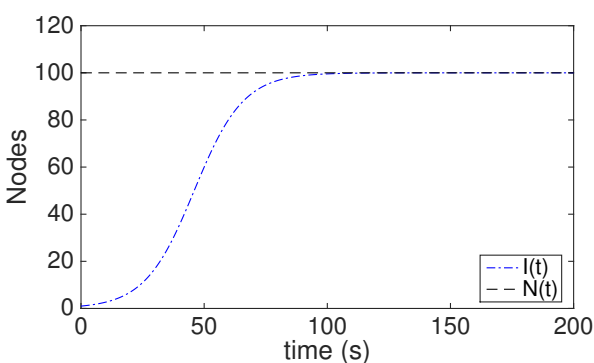

(a)

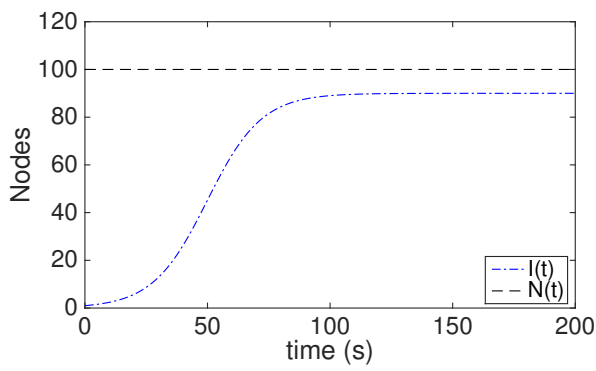

(c)

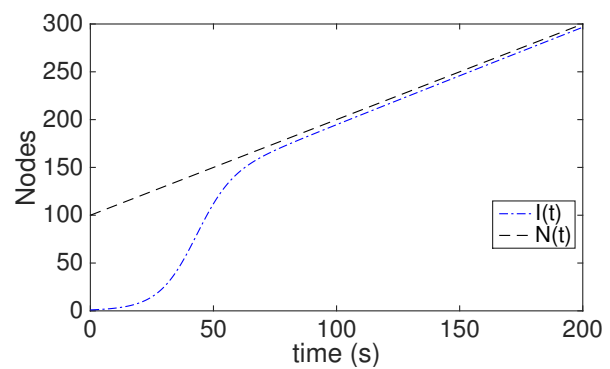

(b)

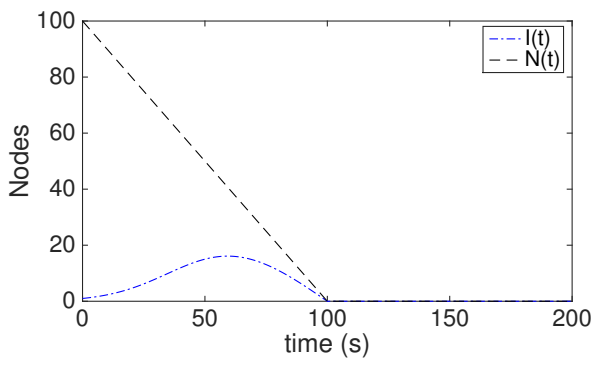

(d)

Figure 1: Evolution of the infected nodes for different values of $\beta$ and $\delta$ : a) $\beta=\delta=0$; b) $\beta=1, \delta=0$; c) $\beta=\delta=1$; d) $\beta=0, \delta=1$

rates. All plots start with the same number of nodes $\left(N_{0}=100\right)$, one infected node $(I(0)=1)$ and contact rate $\lambda=0.001$. Analysing the dynamics of this system, we see that, when there is no arrival and exit rate $(\beta=\delta=0)$ is the basic epidemic model, so the system is stable and all nodes get the message, as we can see in figure $1 \mathrm{a}$. When the system has the same arrival and exit rate (figure $1 \mathrm{c}$ with $\beta=\delta=1$ ), we can see that the system reaches an equilibrium point, but not all the nodes get the message $(I(t)<N(t))$. If $\beta>\delta$, then the number of nodes increases indefinitely as shown in figure $1 \mathrm{~b}$. Finally, when $\beta<\delta$, all the nodes leave the place, and $N(t)$ falls to 0 as shown in figure $1 \mathrm{~d}$

The most relevant case is when incoming and outgoing node rates are equal, that is when $\beta=\delta$. We therefore focus on studying its dynamics and stability in depth. When the system reaches an equilibrium point at time $t_{s}$, this implies that $S(t), I(t), N(t)$ are constant for $t>t_{s}$, so their derivatives are 0 . From equations in (3), this implies, that $N^{\prime}(t)=0=\beta-\delta$, so $\beta=\delta$, and the number of nodes $N(t)=N_{0}$ remains constant to $N_{0}$. In this case, there is a renewal of nodes, with rate $\beta=\delta$. We can obtain an analytical solution for $I(t)$. If we consider the $I^{\prime}(t)$ equation from (3), and replace $N(t)$ with $N_{0}$ and $S(t)$ with $N_{0}-I(t)$, we have:

$$
I^{\prime}(t)=\lambda\left(N_{0}-I(t)\right) I(t)-\beta I(t) / N_{0}=-\lambda I^{2}(t)+\left(\lambda N_{0}-\beta / N_{0}\right) I(t)
$$


The solution of this differential equation when $I(0)=1$ is:

$$
I(t)=\frac{b e^{b t}}{\lambda\left(e^{b t}-1\right)+b} \quad b=\frac{\lambda N_{0}^{2}-\beta}{N_{0}}
$$

Using the expression (5) we can also obtain the number of infected nodes when the system reaches the equilibrium. These equilibrium points can be found assuming the condition $I^{\prime}(t)=0$. Two candidates of equilibrium points are obtained, $I_{e}=\frac{\lambda N_{0}^{2}-\beta}{\lambda N_{0}}$ and $I_{e^{\prime}}=0$. We point out that the number of infected nodes must be always positive $\left(I_{e} \geq 0\right)$, therefore the first point of equilibrium will have sense only if $\lambda N_{0}^{2}-\beta>0$. Since $S(t)+I(t)=N_{0}$ for all $t \geq 0$, from the one-dimensional analysis of the behaviour on the variable $I$, we can directly extend these results to the two-dimensional case. Considering the limits when $t$ tends to $\infty$ in (5), we have that $I(t)$ converges to $\frac{\lambda N_{0}^{2}-\beta}{\lambda N_{0}}$ when $\lambda N^{2}-\beta>0$ and it converges to 0 when $\lambda N^{2}-\beta \leq 0$. As a result, the orbits $(S(t), I(t))$ converge to $\left(N_{0}, 0\right)$ in the case $\lambda N^{2}-\beta \leq 0$ and they converge to:

$$
\left(S_{e}, I_{e}\right)=\left(\frac{\beta}{\lambda N_{0}}, \frac{\lambda N_{0}^{2}-\beta}{\lambda N_{0}}\right)
$$

when $\lambda N_{0}^{2}-\beta>0$. This condition indicates that, when the whole number of contacts ${ }^{3}\left(\lambda N_{0}^{2}\right)$ is greater than the arrival/exit rate $(\beta=\delta)$, the system reaches an equilibrium with $I_{e}$ nodes having the message. Otherwise, the system tends to have zero nodes with the message $\left(I_{e}=0\right)$.

This analytical model allows us to obtain three interesting performance parameters. The first one is the average time a node stays in the area. Using Little's law, $L=\beta W$, where the long-term average number of nodes in a stable system $L$ is equal to to arrival rate multiplied by the average stay (or waiting time) in the system $W$. Thus, in our case, the average stay time is:

$$
W=N_{0} / \beta
$$

The second one is the delivery time $T_{d}$, that is the time when a given number of nodes $M$ get the message. Using equation (5), setting $I(t)=M$ and solving for $t$, we have:

$$
T_{d}(M)=\frac{1}{b} \log \left(\frac{b M-\lambda M}{b-\lambda M}\right) \quad b=\frac{\lambda N_{0}^{2}-\beta}{N_{0}}
$$

Finally, the third one, is the accumulated number of nodes that leave the area with a copy of the message up to time $t$ :

$$
E_{m}(t)=\beta t I_{e} / N_{0}
$$

\footnotetext{
${ }^{3}$ to be precise, the whole number of contacts is $\lambda N(N-1)$, that is practically $\lambda N_{0}^{2}$ when $N$ is large enough.
} 


\subsection{Model considering transmission time}

In this section, we extend the previous model by considering the transmission time of the messages. The message communication time can be expressed as $T_{c}=T_{s}+T_{t}$, and includes two components: a fixed set-up time $T_{s}$ for two nodes to establish a connection; and a transmission time $T_{t}=m / B w$, that depends on the message size $(m)$ and the available bandwidth $(B w)$.

In this model, when an infected node contacts a susceptible node, both nodes establish a connection and a message is transmitted. During the communication time $T_{c}$, the nodes involved cannot infect other nodes, so a new class of nodes is introduced: the communicating $(C)$ nodes. This class is subdivided into two subclasses: subclass $R$, that includes the communicating nodes that are receiving the message, and subclass $P$, that includes the nodes that are transmitting (posting) the message. Thus, when a contact occurs, $R$ and $P$ are increased by one, one coming from the class of infected nodes, and the other one from the class of susceptible nodes, respectively. When transmission ends, these two nodes are moved to the infected nodes class. Note that, in this model, the infected class is defined as the nodes that can infect other nodes (represented by the letter $J$ ).

Additionally, as in the previous model, we have the arrival and exit rates associated with the $S$ and $J$ classes. Nevertheless, the nodes that are transmitting ( $R$ and $P$ classes) are supposed to stay in the place. Thus, the dynamics of this system is expressed through the following transitions (see figure 2):

- $(\rightarrow S, \beta, 0)$ : new nodes arrive in the place with $\beta$ rate.

- $(S \rightarrow R, \lambda S I, 0)$ : a node with no message contacts with a node with the message to start the reception of the message.

- $(S \rightarrow, \beta S /(S+J), 0):$ nodes without the message leave the place.

- $(J \rightarrow P, \lambda S J, 0)$ : a node with the message contacts with a node with no message to start transmission.

- $(S \rightarrow, \delta J /(S+J), 0)$ : nodes with the message leave the place.

- $\left(R \rightarrow J, \lambda S J, T_{c}\right)$ : the reception of the message ends after $T_{c}$ seconds, so now the node its infected.

- $\left(P \rightarrow J, \lambda S J, T_{c}\right)$ : the transmission of the message ends after $T_{c}$ seconds, so the node returns to the infected class.

Note that the third value of the transitions represents the delay incurred, that can be 0 (no delay) or $T_{c}$ (the transmission delay). Using these transitions, we can model the dynamics of this system using the following Delay Differential 


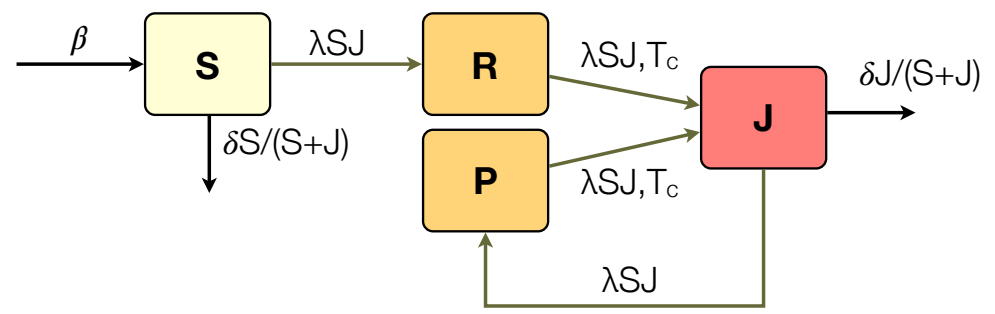

Figure 2: Transitions between classes in an open area with birth and death rates.

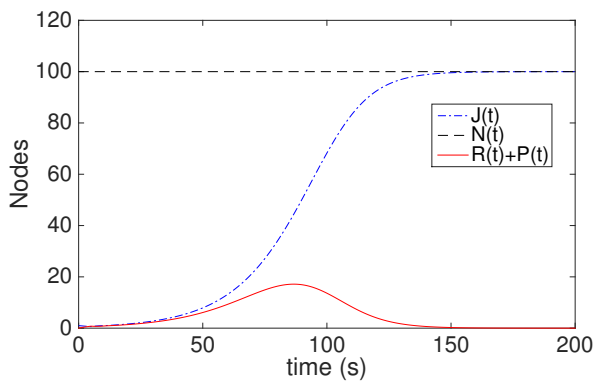

(a)

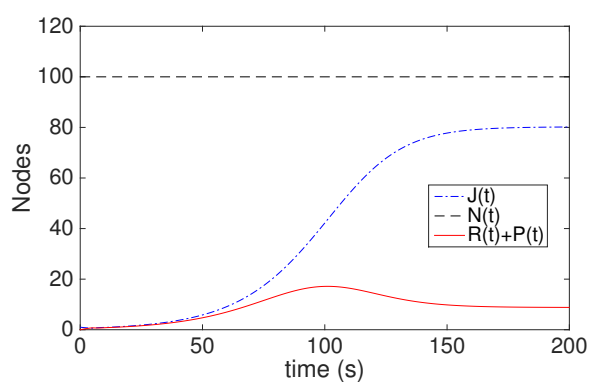

(b)

Figure 3: Evolution of the infected nodes and communicating nodes, with a transmission time $t_{c}=5 s$. a) $\beta=\delta=0$; b) $\beta=\delta=1$;

Equations (DDEs):

$$
\begin{aligned}
S^{\prime}(t) & =-\lambda S(t) J(t)+\beta-\delta S(t) /(S(t)+J(t)) \\
R^{\prime}(t) & =\lambda S(t) J(t)-\lambda S\left(t-T_{c}\right) J\left(t-T_{c}\right) \\
P^{\prime}(t) & =\lambda S(t) J(t)-\lambda S\left(t-T_{c}\right) J\left(t-T_{c}\right) \\
J^{\prime}(t) & =2 \lambda S\left(t-T_{c}\right) J\left(t-T_{c}\right)-\lambda S(t) J(t)-\delta J(t) /(S(t)+J(t)) \\
N^{\prime}(t) & =\beta-\delta
\end{aligned}
$$

with $S(t)=0, J(t)=0 \quad \forall t<0$. As stated previously, $J(t)$ represents the nodes which are able to infect other nodes at time $t$. Since the communicating nodes that are sending the message (class $P$ ) are also infected at time $t$, then the actual number of infected nodes at time $t$ is:

$$
I(t)=J(t)+P(t)
$$

Regarding the dynamics of this system, it is similar to the previous model. When $\beta=\delta$ the system reaches an equilibrium state and the total number ${ }_{230}$ of nodes $N(t)$ remains constant. Figure 3 shows this evolution. We can see, that when no nodes arrive and leave the area, all nodes get infected, and the nodes that are communicating (i.e., $R(t)+P(t)$ ) show an exponential growth up to $t=80 \mathrm{~s}$, and then a fast decrease to zero (when no new nodes can be 
infected). When nodes can arrive and leave the area, the number of infected and communicating nodes are stable for times greater than 180s. As in the previous model, some nodes do not get infected.

When $\beta=\delta$, we can obtain the values of $S_{e}, J_{e}, P_{e}, R_{e}$ which are the fixed (equilibrium) points of the system. The class $P$ (and $R$ ) starts communication with a rate $\lambda S_{e} J_{e}$ and lasts $T_{c}$ seconds, so the number of nodes sending and receiving are:

$$
P_{e}=R_{e}=\lambda S_{e} J_{e} T_{c}
$$

As the number of nodes remains constant, we have that $N_{0}=P_{e}+R_{e}+S_{e}+J_{e}=$ $2 \lambda S_{e} J_{e} T_{c}+S_{e}+J_{e}$. Solving the equation for $J_{e}$ we have:

$$
J_{e}=\frac{N_{0}-S_{e}}{2 \lambda S_{e} T_{c}+1}
$$

Finally, we can obtain the value for $S_{e}$ from the equation of $J^{\prime}(t)$ in $(10)$. For $t$ large enough, we have that $S(t)$ and $J(t)$ are constant, so $S\left(t-T_{c}\right)=S(t)=S_{e}$, $J\left(t-T_{c}\right)=J(t)=J_{s}$ and $J^{\prime}(t)=0$. Then, we have:

$$
0=\lambda S_{e} J_{e}-\delta J_{e} /\left(S_{e}+J_{e}\right)=\lambda S_{e}\left(S_{e}+J_{e}\right)-\delta,
$$

and solving out for $J_{e}$ we have:

$$
J_{e}=\frac{\delta-\lambda S_{e}^{2}}{\lambda S_{e}}
$$

If we equal the right side of this expression with the expression (13), and solving for $S_{e}$, we obtain the following third degree equation:

$$
-2 \lambda^{2} T_{c} S_{e}^{3}+\left(2 \lambda \delta T_{c}-\lambda N_{0}\right) S_{e}+\delta=0
$$

that has three roots. If $2 \delta T_{c}-N_{0} \leq 0$, then $F$ defined as follows:

$$
F\left(S_{e}\right)=-2 \lambda^{2} T_{c} S_{e}^{3}+\left(2 \lambda \delta T_{c}-\lambda N_{0}\right) S_{e}+\delta
$$

that is a strictly decreasing function. Moreover since $\lim _{S_{e} \rightarrow+\infty} F\left(S_{e}\right)=-\infty$ and $\lim _{S_{e} \rightarrow-\infty} F\left(S_{e}\right)=+\infty$ by Rolle's theorem, it only has one real root, that is precisely the solution of $S_{e}$. In this case, due to the complex expression of

fixed point, it is not feasible to apply the same techniques as in the previous model for evaluating the stability of the fixed points. Nevertheless, the empirical evaluation of the system shows the equilibrium of $\left(S_{e}, J_{e}, P_{e}, R_{e}\right)$.

The DDEs of expression (10) does not have a simple analytical solution. Thus, we can solve the DDEs numerically using Euler's method, with a step size of $h$ and time $t_{i}=h i$ :

$$
\begin{aligned}
S_{i+1} & =S_{i}+h\left(-\lambda S_{i} J_{i}+\beta-\delta S_{i} /\left(S_{i}+J_{i}\right)\right) \\
R_{i+1} & =R_{i}+h\left(\lambda S_{i} J_{i}-\lambda S_{i-T_{c}} J_{i-T_{c}}\right) \\
P_{i+1} & =P_{i}+h\left(\lambda S_{i} J_{i}-\lambda S_{i-T_{c}} J_{i-T_{c}}\right) \\
J_{i+1} & =J_{i}+h\left(2 \lambda S_{i-T_{c}} J_{i-T_{c}}-\lambda S_{i} J_{i}-\delta J_{i} /\left(S_{i}+J_{i}\right)\right) \\
N_{i+1} & =N_{i}+h(\beta-\delta)
\end{aligned}
$$


with $R_{i}=0, P_{i}=0, J_{i}=0 \quad \forall i \leq 0, J_{1}=1, N_{0}$ the initial number of nodes, and $t c=\left\lceil T_{c} / h\right\rceil$. We can also obtain the delivery time $T_{d}$ iterating over the 245 previous expression while $\operatorname{round}\left(J_{i}+P_{i}\right)<M$ (that is, while the total number of infected nodes is less than the desired $M$ nodes), so $T_{d}$ will be the time $h i$ when the previous condition is true. We can also obtain the stay time $W$ and the accumulated nodes with message $E_{m}$ using expressions (7) and (9) from the stable fixed point.

250 Studying the effect of the message communication time $T_{c}$, we can see that $I(t)$ is bounded by two components:

1. Contact waiting delay, that is caused by waiting for new contacts. When the communication time is near to zero $\left(T_{c}=0\right)$, all the delay is caused by waiting for contacts so it is equivalent to the basic epidemic model, and $I(t)$ can be approximated with expression (5).

2. Message communication delay, that is caused by the message transmission time. In the absence of contact delay (or when $T_{c}$ is very high), the number of infected nodes doubles after each message transmission with a period $T_{c}$, and can be approximated by the following function:

$$
I_{C}(t)=\min \left(2^{\left\lfloor t / T_{c}\right\rfloor}, N\right)
$$

These components are clearly highlighted in figure 4 , the parameters are the same as in the previous figures, i.e., $N_{0}=100, \lambda=0.001$. Figure 4a shows the results when no nodes arrive and leave the area. For $T_{c}=0$ the diffusion of a message follows the expression (2). For $T_{c}=10$ seconds, it still follows an exponential curve typical of epidemic diffusions. Finally, the curve at the right corresponds to a high transmission time. We can see clearly the effect of the communication time, and how the number of infected nodes doubles approximately every $T_{c}$ seconds $\left(T_{c}=100 s\right)$. This effect is very important because, when the communication time is high, message spreading is delayed by this time and not by the contact rate. In fact, we can see that the number of infected nodes doubles after each period, as represented in expression (18).

Figure $4 \mathrm{~b}$ shows the results when nodes arrive and leave the place. We can see that not all nodes get infected. When $T_{c}=100$, initially, the plot follows the $I_{c}(t)$ curve, but later it exhibits some oscillation, due to this transmission time, that finally vanishes.

Finally, regarding the equivalent continuous Markovian model, the problem is that the inclusion of deterministic delays, makes them non-Markovian. Thus, a more general model is often used, such as the Generalised Semi-Markov Processes (GSMP) 21 or explicit models such as Delayed Continuous-Time Markov Chains [22]. The solution of these models are even more complicated that ithe ones of the non-delayed models. But again, the DDEs model converges to the delayed CTMC, when populations grows, as stated in 21.

A partir de aqui yo lo quitaria y en cada modelo pondria los puntos de equilibrio y las simulaciones, y al final haria una seccion de conclusiones, quitando la seccion de performance evaluation 


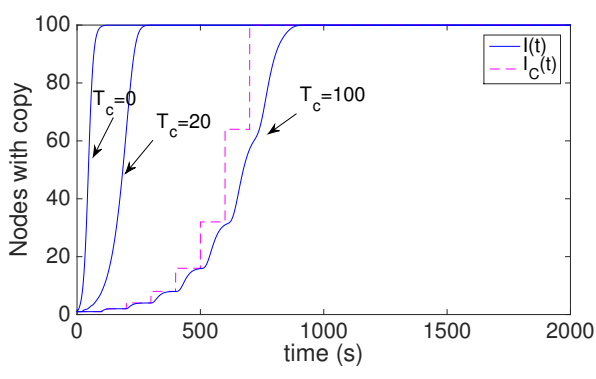

(a)

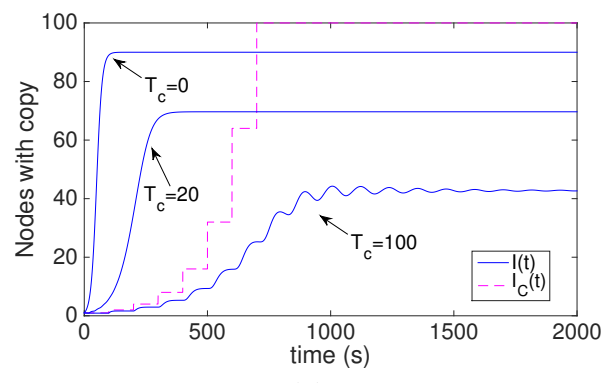

(b)

Figure 4: Evaluation of message diffusion with different transmission times a) $\beta=\delta=0$; b) $\beta=\delta=1$

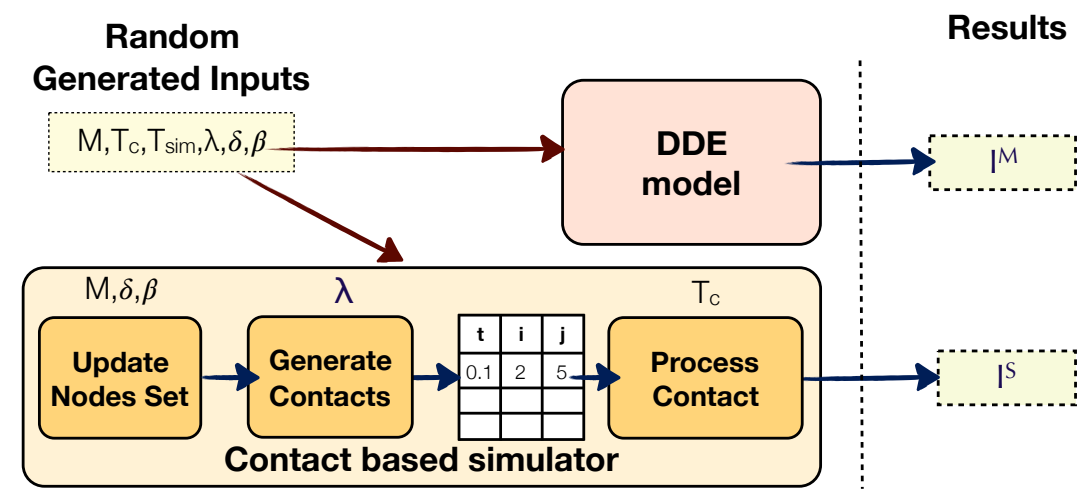

Figure 5: Validation of the model. The input of the simulator and the DDE model are generated randomly. The simulator iteratively is updating the set of nodes and generating contacts between pairs of nodes, in order to simulate the contacts.

\subsection{Model validation}

In this section we detail the procedure we used to validate the models described above, a procedure similar to the one described in [11. The process is depicted in figure 5. We compared the results obtained with the analytical models with those obtained by using a custom simulator that implements the contact-based message diffusion. This simulator is driven by contacts and uses the same parameters of the network model $\left(\lambda, N_{0}, T_{c}, \beta, \delta\right)$. Contacts are generated with an inter-time distribution following an exponential distribution with mean $1 / \lambda$. New nodes without message are created with a $\beta$ rate and nodes are randomly deleted (exit) with rate $\delta S /(S+J)$ for class $S$ and with rate $\delta J /(S+J)$ for class $J$. The simulation computes the number of nodes that have the message up to a given simulation time $T_{\text {sim }}$.

The validation process was based on a set of 1000 random tests (see algorithm in figure 6). Each test adopts different parameter values that are randomly 


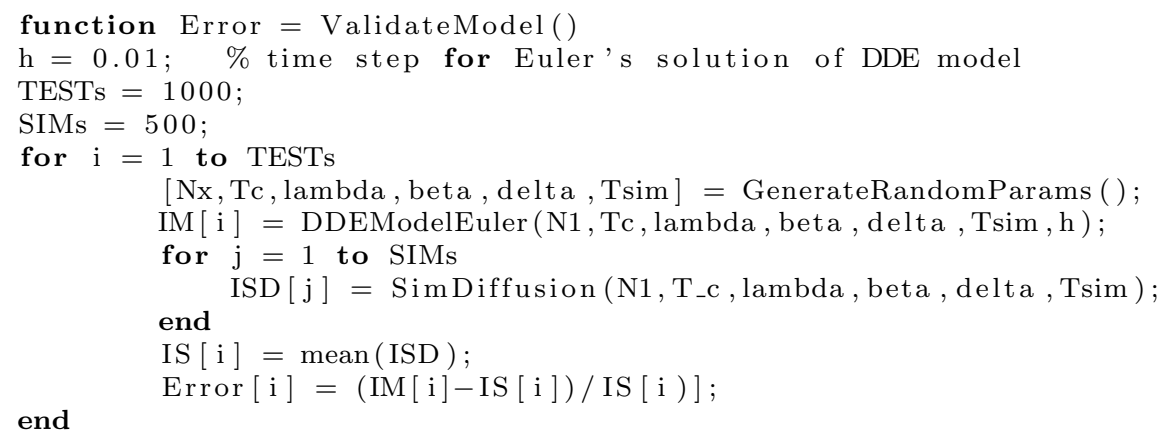

generated from the following defined range of possible values:

$$
\begin{aligned}
& M \sim \mathcal{I}(50,1000) \quad T_{c} \sim \mathcal{U}(0,50) \quad T_{\text {sim }} \sim \mathcal{U}(10,1000) \\
& \lambda \sim \mathcal{U}(0.001,0.1) \quad \delta \sim \mathcal{U}(0.001,0.1) \quad \beta \sim \mathcal{U}(0,10)
\end{aligned}
$$

where $\mathcal{U}(a, b)$ stands for the uniform distribution (over interval $(a, b))$ and $\mathcal{I}(a, b)$ for man uniform integer distribution.

For each test $i$, the test program generates randomly the parameters to test and first obtains $I(t)$ from our model $\left(I_{i}^{M}\right.$, that is IM [i] in code), using the Euler's method (function DDEModelEuler) with $h=0.01$. Using the same parameters, the simulation is repeated 500 times (function SimDiffusion), generating for each simulation contacts with a rate $\lambda$ up to time $T_{\text {sim }}$ in order to obtain a mean of the number of infected nodes $\left(\overline{I_{i}^{S}}\right.$, that is IS [i] in code). Note, that each simulation represents a realisation of the process and it depends clearly on the distribution of the contacts, so this must be repeated. That is, in each simulation using the same contact rate $\lambda$ and arrival/exit rates $(\beta, \delta)$ different sequences of contacts are generated. Finally, the modelling error is obtained as follows:

$$
\epsilon_{i}=\frac{I_{i}^{M}-\overline{I_{i}^{S}}}{\overline{I_{i}^{S}}}
$$

After running 1000 tests we obtained the mean error and $95 \%$ confidence inter300 vals: $\bar{\epsilon}=0.71(0.15-1.53) \%$. These results validate the models proposed in this paper.

\section{Perfomance evaluation}

The models introduced in section 3 allow us to evaluate the dynamics of the diffusion of messages in an area and, when the system reaches an equilibrium 305 point, parameters such as the number of infected nodes, the diffusion time and the waiting time. Since the evolution and dynamics of the system were already 
studied in section 3 , in this section we focus our evaluation assuming that the system reaches an equilibrium state, that is, assuming that the arrival and exit rates are the same. From now on, we refer to both rates jointly as the renewal

We consider an area with $N_{0}$ initial individuals, that can move freely, entering and leaving the place with a renewal rate $\beta=\delta$ and carrying a mobile device that can establish pair-to-pair connection using Bluetooth (more exactly a Bluetooth 2.0, Class 2 device). The rate of contacts $\lambda$ can be approximated using the expression given in 5 . We consider a communication range of $7.5 \mathrm{~m}$ (an average bluetooth range), an area of about $100 \mathrm{~m}^{2}$ and a mean speed of nodes of $0.5 \mathrm{~m} / \mathrm{s}$, a combination of values that gives a value of $\lambda=0.001 \mathrm{~s}^{-1}$, that is, a pairwise contact rate of about 3.6 contacts/h. Regarding to the communication time, we used a mean bandwidth of $B w=2.1 \mathrm{Mb} / \mathrm{s}$ and a setup time $T_{s}=0.1 s$.

The goal of the first experiment is to evaluate the delivery time of a message. We first assume that all nodes stay inside the area (that is, there is no renewal rate, so $\beta=\delta=0$ ), so all nodes can receive the message. The number of nodes ranges from 10 to 2000, corresponding to an average density ${ }^{4}$ of 0.001 to 0.2 people per square meter $\left(p / m^{2}\right)$. We used three different message sizes: a short text message $(m=1 \mathrm{~KB})$, that has a very short communication time $\left(T_{c}=0.1 s\right)$, a typical image message $(m=1 \mathrm{MB})$, with a communication time $T_{c}=4.3 \mathrm{~s}$, and finally a short video $(m=10 \mathrm{MB})$ with $T_{c}=42 \mathrm{~s}$. We can see in figure $7 \mathrm{a}$, that for very low densities, the delivery time is very high, although this delivery time decreases exponentially with density of people up to to $0.01 \mathrm{p} / \mathrm{m}^{2}$. For higher densities, the delivery time decreases very slowly, so the epidemic diffusion for higher people densities does not produce a significant improvement. For $m=10 \mathrm{MB}$ (a diffusion bounded by the message communication delay), we can see that the delivery time is higher, and it does not decrease with the number of nodes; in fact, it slightly increases with the number of nodes, due to the increase of the message communication delay component.

We now evaluate the delivery time with renewal rate and no transmission delay using expression (8). Figure $7 \mathrm{~b}$ shows the delivery time when the message is received for some percentage of the nodes (that is, $100 \cdot M / N_{0}$ ), when the renewal rate is 5 . We can clearly see that the delivery time decreases exponentially with the density. Figure $7 \mathrm{c}$ shows the delivery time to $90 \%$ of the nodes for a renewal rate of 5, and with different message sizes. Comparing these results with the ones with no renewal rate in figure $7 \mathrm{a}$ we observe that the pattern is very similar: in general with less time (note that this delivery time corresponds to $90 \%$ of nodes), but when people density is low, the delivery time reaches $\infty$ (that is, less than $90 \%$ of nodes receive the message). In this graph we can see a interesting effect: for $m=1 \mathrm{~KB}$, when density is less than 0.02 , the delivery time is $\infty$, that is the message is not delivered; however, for large message sizes

\footnotetext{
${ }^{4}$ In order to make the experiments independent of the nodes and place area, we chose to use people density in the graphs
} 


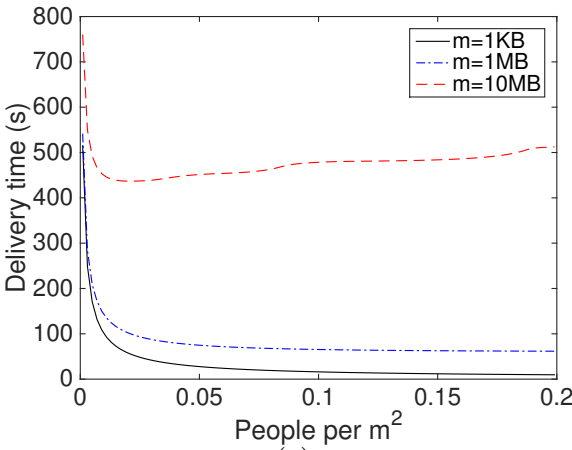

(a)

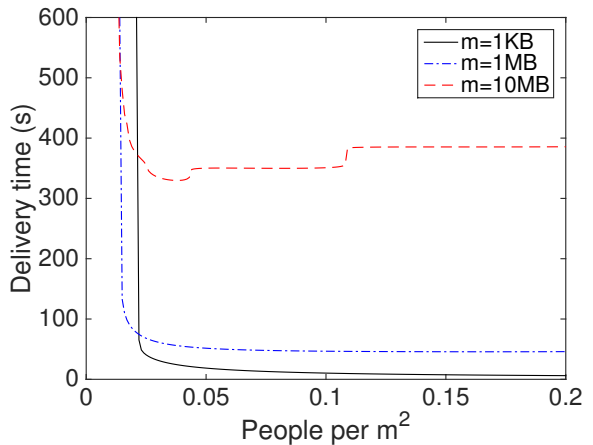

(c)

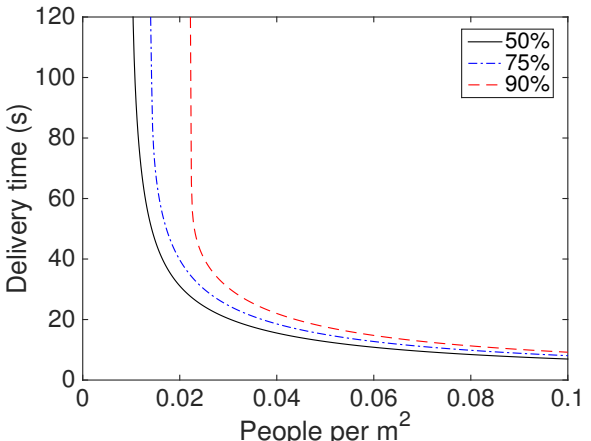

(b)

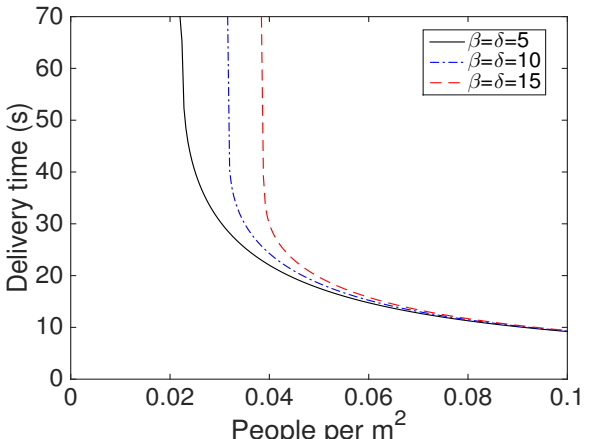

(d)

Figure 7: Delivery time depending on the people density. a) Results of different message sizes with no renewal rate. b) With a renewal rate of 5 and no transmission delay for different percentages. c) Results of the delivery time to $90 \%$ of nodes with a renewal rate of 5. d) Delivery time to $90 \%$ of the nodes with different renewal rate and no transmission delay

( $m=1 \mathrm{MB}$ and $m=10 \mathrm{MB}$ ) although the delivery time is higher, it can be deliv-

ered. The reason of this behaviour, is that the nodes which are communicating cannot leave the system, so, if the transmission time is higher, then the ratio $J(t) /(S(t)+J(t))$ is lower, so, more nodes with the message stay in the place. Finally, in figure $7 \mathrm{~d}$, we evaluate the impact of different renewal rates, obtaining the delivery time when the message is received by the $90 \%$ of nodes. We can see that higher renewal rate implies that the delivery time increases exponentially when the number of nodes decreases, reaching $\infty$ (that is, the message is not delivered to the $90 \%$ of nodes).

Considering the effectiveness of the diffusion, that is, the final percentage of nodes that receive the message $\left(100 \cdot I_{e} / N_{0}\right)$, we observe that with no renewal

360 rate, the effectiveness of the diffusion is $100 \%$, although, as shown in figure $7 \mathrm{a}$, when the density is very low, the delivery time is very high. Figure 8 a shows this percentage depending on the density of people and for a fixed renewal rate of 5 persons per second with different messages sizes. We can see that for densities below $0.01 \mathrm{p} / \mathrm{m}^{2}$ the percentage of nodes that receive the message is very low, due to the effect of the renewal rate. We can also see that for a larger message 


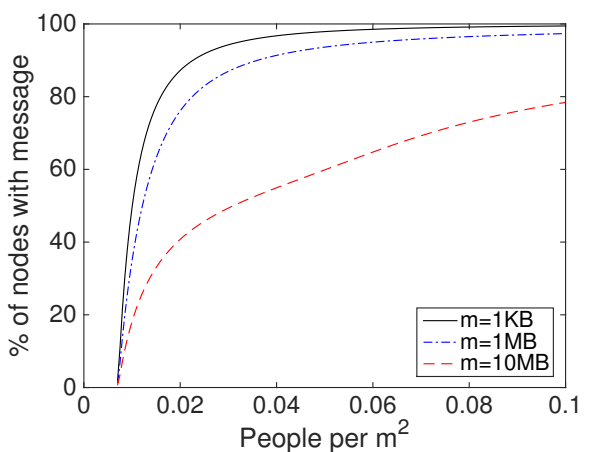

(a)

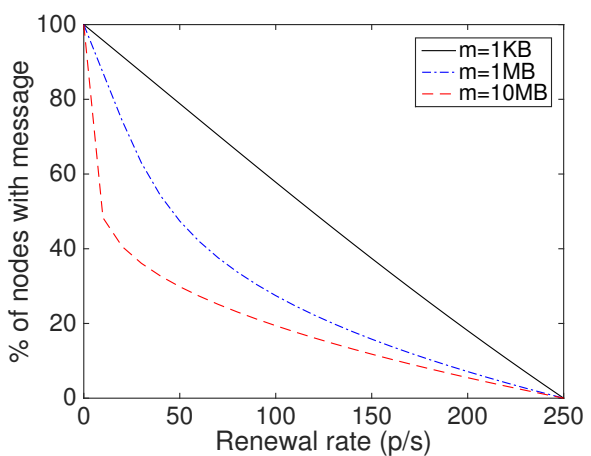

(b)

Figure 8: Ratio of nodes with a message. a) Depending on density with a renewal rate $\beta=\delta=5$; b) Depending on the renewal rate for a density of $0.05 p / \mathrm{m}^{2}$.
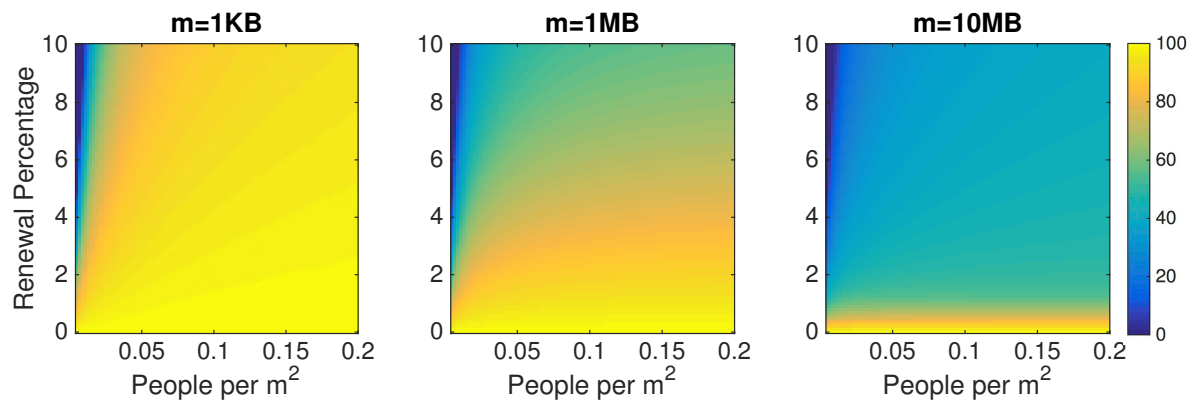

Figure 9: Effectiveness of the diffusion depending on people density and renewal ratio for different message sizes.

size (that is, a large communication time), the percentage is very low, because it takes more time to transmit a message. When the number of nodes increases the effect of the fixed renewal rate is reduced, reaching practically $100 \%$ of nodes when density is very high. Figure $8 \mathrm{~b}$ shows the ratio depending on the renewal rate for a fixed density of $0.05 \mathrm{p} / \mathrm{m}^{2}\left(N_{0}=500\right)$. We can observe that for short messages $(m=1 K B)$ the decrease of the ratio is almost lineal with the renewal rate, but for larger messages and short renewal rates this ratio decreases quickly.

The results of the previous evaluations reveal that the factors which have more impact on the effectiveness of the diffusion are people density, renewal rate 375 and message size. Figure 9 includes three density plots of the percentage of nodes that receive the message depending of people density and renewal rati 5 . These plots give a clear vision of the impact of the different factors. For $m=1 \mathrm{~KB}$, we can see that effectiveness is very high (greater than $80 \%$ ). Nevertheless, for

\footnotetext{
${ }^{5}$ In these plots we use the renewal ratio $(R R)$, that is, the percentage of nodes that are renewed in the place every second $\left(\beta=\delta=R R / 100 \cdot N_{0}\right)$
} 
larger message sizes, the effectiveness is reduced specifically when the renewal

380

We repeated the previous experiments using other network parameters, such as place size, node speed, etc. From these experiments, we can say that the main effect of varying $\lambda$ is the diffusion time and not the pattern of the diffusion, and the same conclusions can be extracted from these experiments. Specially, the 385 results shown in figure 9 are equivalent using different places sizes, confirming the impact of people density and renewal percentage.

\section{Conclusions}

In this paper, we analytically modelled the performance of mobile opportunistic networks for contact-based messaging applications in city squares or gathering points, a key topic necessary for the effective design of novel services. In our study we took into account several social aspects such as: the density of people, the dynamic of people arriving and leaving a place, the size of the messages and the duration of the contacts.

We first introduced a dynamical model that takes into account that nodes can arrive and leave the area. For this model we studied the stability of the fixed points and obtained analytical expressions for its resolution. We proved that when the system reaches the equilibrium, the arrival and exit rates are the same, so the total number of nodes in the area remains constant, but this renewal rate implies that not all nodes receive the message. We extended this model 400 taking into account the communication time for transmitting the message. This model describes a system that is also stable when the arrival rate equals the exit rate. For this model, we also obtained the analytical expressions for its fixed points and the number of nodes that get the message. The evaluations performed showed that the models can reproduce the dynamics and stable states of message diffusion.

Several important conclusions are derived from the evaluations detailed in the paper. As expected, when people density increases, the effectiveness of the diffusion is improved (that is, the delivery time is reduced and the percentage of nodes that receive the message increases). Regarding the renewal of nodes, its impact is more important when people density is low. And finally, message size has a strong impact on message diffusion. For large message sizes the effectiveness of the epidemic diffusion is reduced. This is specially important when the renewal rate is slightly increased.

The applications of these models are very important in the design and de415 ployment of message diffusion systems. For example, if the model predicts a low effectiveness of the diffusion in a given are, communication enablers like data repeaters can be deployed to increase the effectiveness of diffusion. 


\section{References}

[1] Y. Wang, A. Vasilakos, Q. Jin, J. Ma, Survey on mobile social networking in proximity (MSNP): approaches, challenges and architecture, Wireless Networks 20 (6) (2014) 1295-1311.

[2] E. Toledano, D. Sawada, A. Lippman, H. Holtzman, F. Casalegno, Cocam: Real-time photo sharing based on opportunistic p2p networking, in: Consumer Communications and Networking Conference (CCNC), 2013 IEEE, 2013, pp. $877-878$.

[3] J. Su, J. Scott, P. Hui, J. Crowcroft, E. de Lara, C. Diot, A. Goel, M. Lim, E. Upton, Haggle: Seamless networking for mobile applications, in: J. Krumm, G. Abowd, A. Seneviratne, T. Strang (Eds.), UbiComp 2007: Ubiquitous Computing, Vol. 4717 of Lecture Notes in Computer Science, Springer Berlin Heidelberg, 2007, pp. 391-408.

[4] M. Khabbaz, C. Assi, W. Fawaz, Disruption-tolerant networking: A comprehensive survey on recent developments and persisting challenges, Communications Surveys Tutorials, IEEE 14 (2) (2012) 607-640.

[5] R. Groenevelt, P. Nain, G. Koole, The message delay in mobile ad hoc networks, Performance Evaluation 62 (2005) 210-228.

[6] Z. J. Haas, T. Small, A new networking model for biological applications of ad hoc sensor networks, Networking, IEEE/ACM Transactions on 14 (1) (2006) 27-40.

[7] T. Spyropoulos, K. Psounis, C. Raghavendra, Efficient routing in intermittently connected mobile networks: The multiple-copy case, Networking, IEEE/ACM Transactions on 16 (1) (2008) 77 -90.

[8] E. Hernandez-Orallo, M. Serrat Olmos, J.-C. Cano, C. Calafate, P. Manzoni, CoCoWa: A collaborative contact-based watchdog for detecting selfish nodes, Mobile Computing, IEEE Transactions on 14 (6) (2015) 1162-1175.

[9] M. Karaliopoulos, Assessing the vulnerability of DTN data relaying schemes to node selfishness, Communications Letters, IEEE 13 (12) (2009) $923-925$.

[10] J. Whitbeck, V. Conan, M. Dias de Amorim, Performance of opportunistic epidemic routing on edge-markovian dynamic graphs, Communications, IEEE Transactions on 59 (5) (2011) 1259-1263.

[11] X. Zhang, G. Neglia, J. Kurose, D. Towsley, Performance modeling of epidemic routing, Computer Networks 51 (10) (2007) 2867 - 2891.

[12] C. S. De Abreu, R. M. Salles, Modeling message diffusion in epidemical DTN, Ad Hoc Networks 16 (2014) 197-209. 
[13] Q. Xu, Z. Su, K. Zhang, P. Ren, X. S. Shen, Epidemic information dissemination in mobile social networks with opportunistic links, Emerging Topics in Computing, IEEE Transactions on 3 (3) (2015) 399-409.

[14] T. G. Kurtz, Approximation of Population Processes, SIAM, 1981.

[15] Y. Kuang, Delay Differential Equations: With Applications in Population Dynamics, Mathematics in Science and Engineering, Elsevier Science, 1993.

[16] M. Alizadeh, A. Javanmard, B. Prabhakar, Analysis of DCTCP: Stability, convergence, and fairness, in: Proceedings of the ACM SIGMETRICS Joint International Conference on Measurement and Modeling of Computer Systems, SIGMETRICS '11, ACM, New York, NY, USA, 2011, pp. 73-84.

[17] A. Lindgren, A. Doria, O. Schelén, Probabilistic routing in intermittently connected networks, SIGMOBILE Mob. Comput. Commun. Rev. 7 (3) (2003) 19-20.

[18] T. Spyropoulos, K. Psounis, C. S. Raghavendra, Spray and wait: An efficient routing scheme for intermittently connected mobile networks, in: Proceedings of the 2005 ACM SIGCOMM Workshop on Delay-tolerant Networking, WDTN '05, ACM, New York, NY, USA, 2005, pp. 252-259.

[19] L. J. S. Allen, Mathematical Epidemiology: Lecture Notes in Mathematics, Vol. 1945, Springer Verlag, 2008, Ch. An Introduction to Stochastic Epidemic Models, pp. 81-130.

[20] T. G. Kurtz, Solutions of ordinary differential equations as limits of pure jump markov processes, Journal of Applied Probability 7 (1) (1970) 49-58.

[21] L. Bortolussi, J. Hillston, Fluid approximation of ctmc with deterministic delays, in: Quantitative Evaluation of Systems (QEST), 2012 Ninth International Conference on, 2012, pp. 53-62.

[22] C. Guet, A. Gupta, T. Henzinger, M. Mateescu, A. Sezgin, Delayed continuous-time markov chains for genetic regulatory circuits, in: P. Madhusudan, S. Seshia (Eds.), Computer Aided Verification, Vol. 7358 of Lecture Notes in Computer Science, Springer Berlin Heidelberg, 2012, pp. 294-309. 